\title{
Piezoelectric polymer composites for sensors and actuators
}

\author{
E. Carvalho ${ }^{1,2}$, L. Fernandes ${ }^{1,3}$, C. M. Costa ${ }^{1,2}$, S. Lanceros-Méndez $z^{3,4}$ \\ ${ }^{1}$ Centro de Física, Universidade do Minho, 4710-057 Braga, Portugal \\ ${ }^{2}$ Institute of Science and Innovation for Bio-Sustainability (IB-S), University of Minho, \\ Portugal \\ ${ }^{3}$ BCMaterials, Basque Center for Materials, Applications and Nanostructures, UPV/EHU \\ Science Park, 48940 Leioa, Spain \\ ${ }^{4}$ IKERBASQUE, Basque Foundation for Science, 48013, Bilbao, Spain
}

\begin{abstract}
:
As a result of the Internet of Things (IoT) and Industry 4.0 paradigms, based on increasing interconnectivity, the development of advanced high-performance materials for sensor and actuator applications are increasingly required. In particular, piezoelectric composites are of large scientific and technological interest from fundamental and applied point of views. Piezoelectric composites are applied in a wide range of applications as they combine the excellent properties of polymers and ceramics. The definition and properties of piezoelectric materials and composites are presented as well as the recent applications in areas such as electronics, energy harvesting, environmental sensors and biomedical applications. The outlook and future trends for piezoelectric composites are also provided.
\end{abstract}

Keywords: piezoelectric; sensors; actuators; biomedical applications; dielectric constant; composites; multifunctional materials; smart materials. 


\section{Introduction}

Currently, with the Internet of Things (IoT) and Industry 4.0 paradigms, increasingly requiring smart and multifunctional materials with higher performance, piezoelectric composites are gathering particular attention, as they can be applied in a wide range of applications from sensors and actuators to biomedical applications, being processable by conventional and additive manufacturing techniques [1].

Polymer composites result from the combination of a polymeric matrix and different fillers (one or two different fillers with complementary properties), gathering the advantages of the polymeric matrix (low density and flexibility) and the fillers (mechanical and thermal properties, or increased functional response) [2].

In relation to fillers, they can be conductive [3], magnetic [4] and ceramic [5], ceramic fillers having as main advantages the possibility of being piezoelectric with high piezoelectric coefficients, low dielectric and mechanical losses, and wide variety of dielectric constants [6]. It is important to notice that the manufacturing method, the particle size and the dispersion method play an essential role in the final properties of piezoelectric polymer composites [7].

In addition, piezoelectric polymer composites can be particulate [8] and/or laminate [9] composites and the dispersion of each component is defined by the connectivity, that designates the interconnection of the different phases of the composite materials[10].

The connectivity influences the final structure of the piezoelectric polymer composite, which in turn influences the macroscopic response and, therefore, the application possibilities [11].

One of the most recent trends in piezoelectric composites is the production of these materials with two different fillers, such as ceramic and conductive fillers [12], ceramic and magnetic fillers [13] and combinations thereof, such as core-shell fillers [14], in order to improve performance or to provide multifunctionality for applications in areas such as dielectric-based capacitors, batteries, electronic devices and microwave absorption devices.

In the following, the main definitions and properties in relation to polymer composites will be presented, as well as recent advances divided by application. In addition, the main materials for polymers and fillers will be presented. 


\section{Piezoelectric Sensors and actuators: definition and properties}

The name Piezoelectricity, was proposed by Hankel in 1881, and signifies "electricity by pressure" being derived from the Greek word piezo which means pressure [15]. However, the concept of piezoelectricity was discovered a year before, in 1880, by the Curie brothers where it was found that mechanical stresses induced macroscopic polarization, i.e. the generation of electric surface charges, in several crystals such as zincblende, topaz and quartz [16]. The converse piezoelectric effect was predicted only a year later by Lippmann, derived from the thermodynamic theory, where an external electric potential is capable of producing mechanical deformations/strains to the materials [17]. With these initial discoveries, a large interest was paid in this class of materials due to their applicability in areas ranging from sonars to microphones, accelerometers and pressure transducers, among others [18].

Another breakthrough was achieved in 1969 by Kawai with the discovery of a strong piezoelectric effect in poly(vinylidene fluoride) (PVDF) adding mechanically flexible materials to the list of piezoelectric materials [19]. The discovery of these flexible piezoelectric materials extended the range of applications to flexible electronics, large area sensors, flexible energy harvesters and biomedicine.

By definition, piezoelectric materials, a class of dielectric materials, are a family of both inorganic and organic materials upon which polarization can be varied by the application of a mechanical stress, or vice versa, as represented in Figure 1. They can be divided into two classes, namely polar and non-polar piezoelectric materials, depending on the existence of a net dipole moment or a null total dipole moment respectively. 


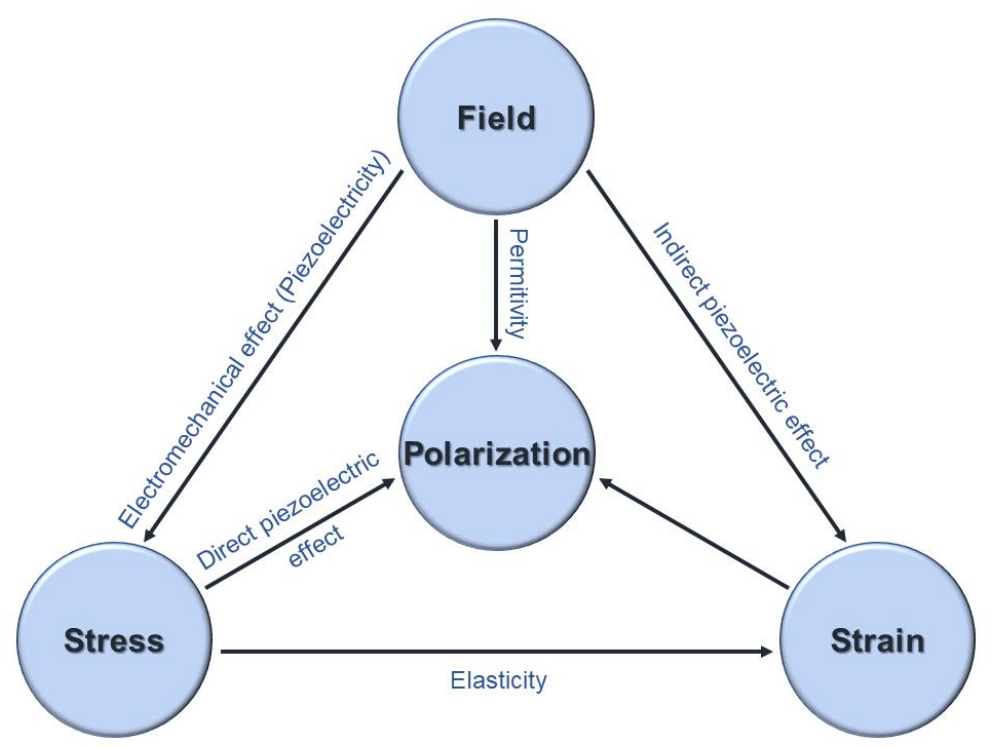

Figure 1 - Schematic representation of the sources of the piezoelectric phenomena. Adapted from [20].

In Figure 2 it is shown a simplified molecular model explaining the behavior of a piezoelectric material. Prior to exerting an external stress to the material, in each molecule, the centers of the positive and negative charges coincide (Figure 2a)), i.e. the external effects of the charges are equally null, and the molecule is electrically neutral. Upon application of an external mechanical stress to the material, deformation of the internal structure of the molecule occurs and, as a result of the separation of the positive and negative gravity centers, dipoles are generated (Figure 2b)). The opposite facing poles in the material are mutually canceled and fixed charges appear on its surface (Figure 2c)) [21]. This is known as the direct piezoelectric effect, schematized in Figure 1 [20].

A year after the discovery of the piezoelectric effect, in 1881, the Curie brothers proved the theory developed by Lippmann showing that piezoelectric materials may also have the reverse behavior, i.e. when an electric potential is applied across the electrodes, a mechanical deformation/strain occurs [17]. For this case it is said that the materials have a reverse piezoelectric effect [21]. Materials that present this behavior can be implemented in several applications as actuators or positioning devices [20]. 


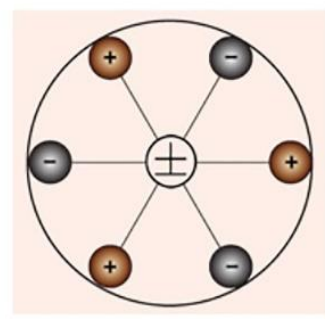

(a)

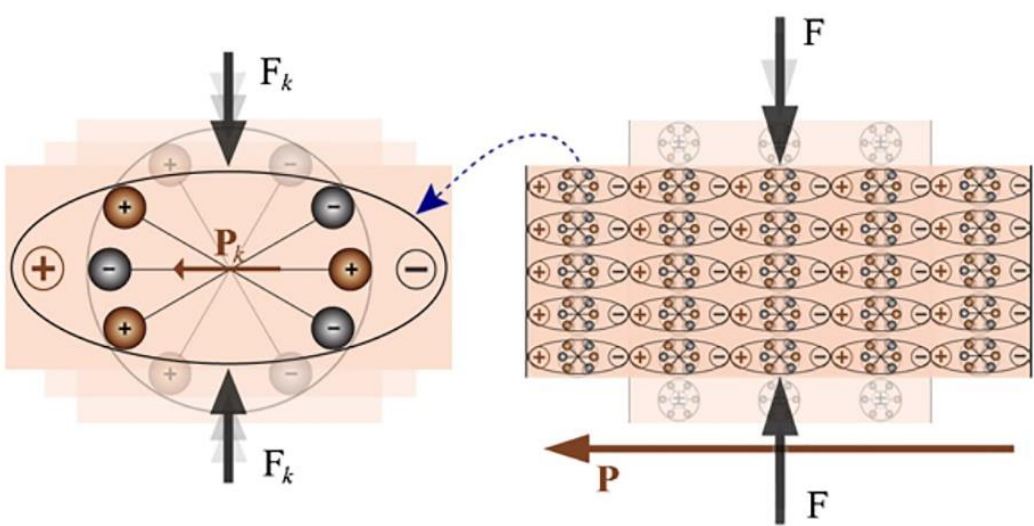

(b)

(c)

Figure 2 - Schematic representation of the piezoelectric effect: a) electrically polarized unperturbed molecule, b) application of an external force $\left(\mathrm{F}_{\mathrm{k}}\right)$ and induced polarization $\left(\mathrm{P}_{\mathrm{k}}\right)$ and $\left.\mathbf{c}\right)$ polarizing effect on the surface of the piezoelectric material [20].

A schematic representation of the behavior of piezoelectric materials containing two metal electrodes deposited on the surfaces where the opposite surface charges are formed is represented in Figure 3. If the electrodes are short circuited with a galvanometer connected to the wire, when pressure is applied to the piezoelectric material a charge density appears on the surfaces of the crystal in contact with the electrodes. The free charges will move until they neutralize the polarization effects as presented in Figure 3a). Once this external force is removed, the polarization disappears with the flow of the free charges reversing and the material returning to its original state (Figure 3b)) [21]. On the other hand, by replacing the short-circuiting wire with a resistance, the current would be capable of flowing through, thus converting mechanical energy into electrical energy, which is the basis of energy harvesting applications [22]. 


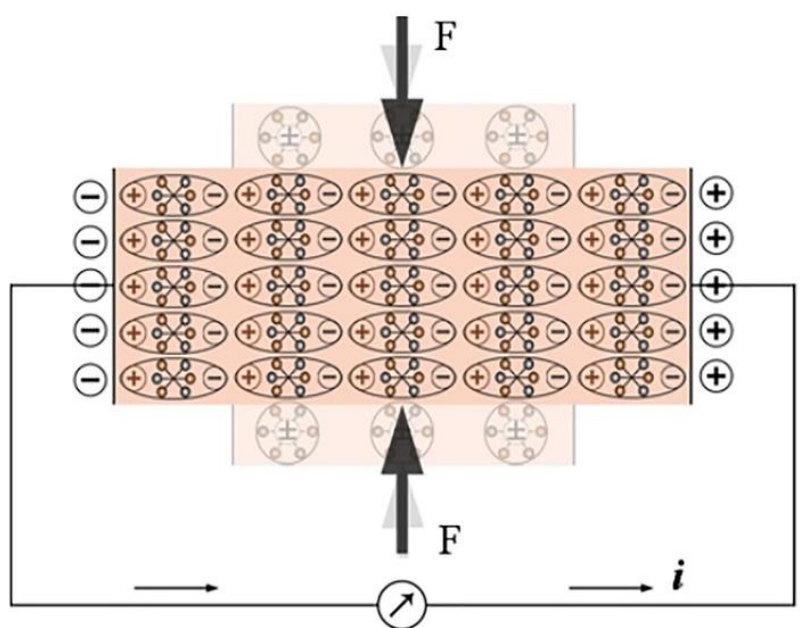

(a)

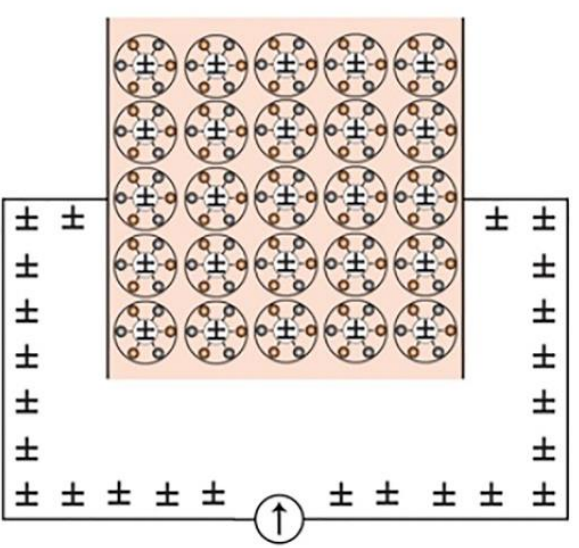

(b)

Figure 3 - Representation of the piezoelectric phenomena: a) neutralizing current flow of a piezoelectric material with two short circuited terminal subjected to an external force and b) material in its original state with the absence of current in the short-circuit [20].

Being anisotropic by nature, piezoelectric materials mechanical, electrical and electrochemical properties vary depending on the direction of the mechanical and/or electrical stimuli and thus the implementation of such materials in the sensing and actuating areas require an in-depth evaluation of the magnitude of the various properties in the different directions [23].

In most of the cases, the material must undergo a poling process in order to properly orient the dipoles and, thus, maximize the piezoelectric response. This poling is typically achieved by the application of and electrical field along a specific direction. Posteriorly, a smaller electric potential can be applied to the materials inducing changes in its dimensions. If this electric potential has the same direction as the poling field, additional expansion on the poling direction and contraction perpendicularly occurs [20]. 

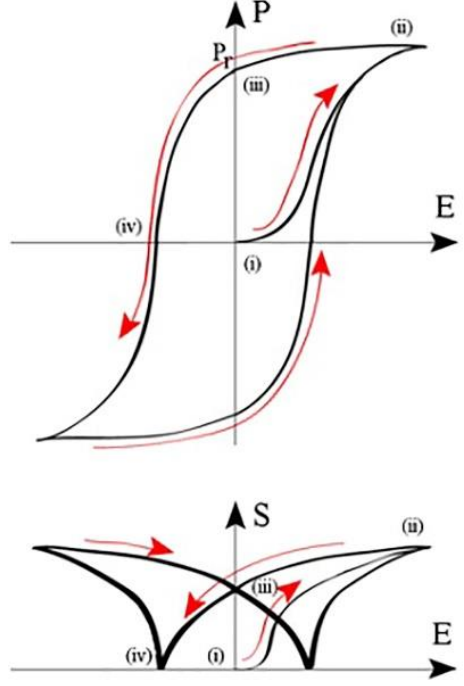

(a)

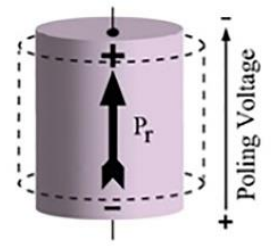

(b)

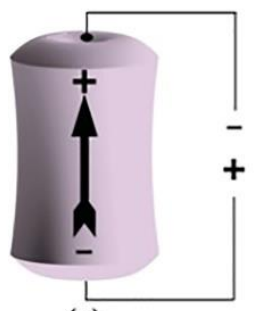

(c)

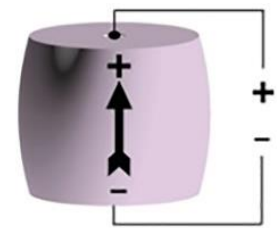

(d)

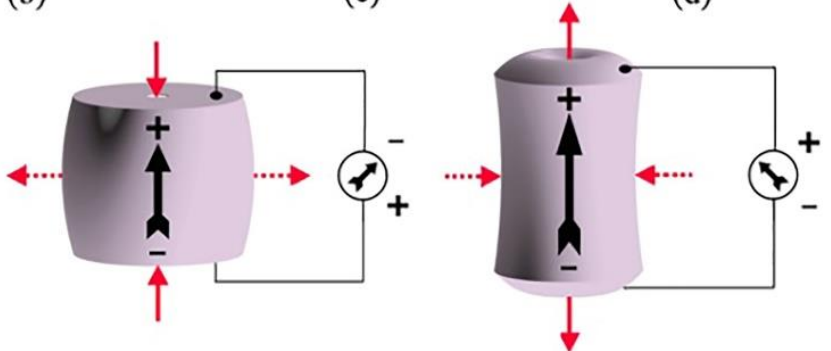

(e)

(f)

Figure 4 - Behavior of a piezoelectric material as sensor and actuator. (a) Typical P-E hysteresis and S-E plots. (b) The piezoelectric material before (dotted) and after poling, the polarity of poling field is indicated. (c) Mechanical deformation when the applied electric potential has polarity similar to the poling field and (d) when the applied electric potential has opposite polarity to the poling field. (e) Generated electric potential with polarity similar to poling field when compressive force is applied in the same direction and (f) with polarity opposite to poling field when tensile force is applied in poling direction [20].

In Figure 4a are represented the typical polarization vs electric field (P-E) hysteresis and strain vs electric field (S-E) plots of a piezoelectric material. With the application of an electric field across a piezoelectric material, both the polarization and the strain curves, in the P-E and S-E plots respectively, follow the path (i) to (ii). Once this field is removed, a remnant polarization $\left(P_{r}\right)$ and a permanent change in the dimensions is experience by the material, shown in the curves as the path (ii) to (iii), and a working point shift from the material occurs the plots in Figure 4a). After this, two situations can occur. First, if an electric potential with the same polarity as the poling field is applied, the plots if Figure 4a) follow the path (iii) to (ii). Meaning that the piezoelectric material will experience and expansion along the poling axis (Figure 4c)). On the other hand, if the applied electric potential has the opposite polarity as the poling field, the P-E and S-E plots will follow the path (iii) to (iv) and the material experiences a contraction along the poling field axis and expansion perpendicularly to it (Figure 4d)). For both situations, the piezoelectric 
material, when the electric potential is removed, returns to the poling dimensions (iii) in the plots [20].

Similarly, for the reverse piezoelectric effect, an electric potential is generated when a tensile force is applied (Figure 4e) and f)). In Figure 4e) it is shown that if a compressive force is applied along or tensile force perpendicular to the poling axis, the generated electric potential will have the same polarity as the poling axis. Nevertheless, if a compressive force is applied perpendicularly or tensile force is applied parallel to the poling axis, the generated electric potential will have and opposite polarity to the poling axis (Figure 4f)) [20].

From a mathematical point of view, when low electric fields and/or low mechanical stress are applied, piezoelectric materials show a linear response [24]. When stress is applied to a piezoelectric material, there will be a variation of the electrical polarization and, as a consequence, electric charge will be produced on the materials surface. Thus,

$$
P_{p e}=d . T
$$

where $P_{p e}$ is the piezoelectric polarization vector, $d$ is the piezoelectric strain coefficient and $T$ is the stress subjected to the material. Similarly, the reverse piezoelectric effect can be expressed by means of

$$
S_{p e}=d . E
$$

with $S_{p e}$ being the produced mechanical strain and E the magnitude of the applied electric field. Taking into account the piezoelectric materials elastic properties, the piezoelectric effect can be formulated as

$$
\begin{gathered}
P_{p e}=d . T=d . s . T=e . S \\
T_{p e}=\text { c. } S_{p e}=d . c . E=e . E
\end{gathered}
$$

with $c$ being the elastic constant (which relates the generated stress and applied strain as $T=c \times S$ ), $s$ being the compliance coefficient (relating the produced deformation with the applied stress as $S=s \times T$ ) and $e$ being the piezoelectric stress constant.

When the piezoelectric material is subjected to a strain, this has two implications. First, an electric polarization variation is generated and, for the other, an elastic stress $T_{e}$ occurs. Additionally, the generated electrical polarization variation leads to an internal electric field $E_{p e}$ variation which can be written as 


$$
E_{p e}=\frac{P_{p e}}{\varepsilon}=\frac{e . S}{\varepsilon}
$$

with $\varepsilon$ being the materials dielectric constant. The application of a compressive stress in the same direction of the polarization direction will induce an electric field variation with the same polarity. Moreover, the presence of an electric field in polarization direction results in an expansion of the piezoelectric material in the same direction (Figure 4d)). This means that the directions of the produced and applied stresses are opposite, which is equivalent if the nature of the applied stress is tensile. This means that the produced stress $T_{p e}$ is opposite to the piezoelectric material's deformation and, by consequence the stress generated can be written as

$$
T=T_{e}+T_{p e}=c . S+\frac{e^{2}}{\varepsilon} \cdot S=\left(c+\frac{e^{2}}{\varepsilon}\right) \cdot S=\bar{c} . S
$$

with $\bar{c}$ being the piezoelectric stiffened constant. Hence, in the presence of the piezoelectric effect the material becomes more rigid.

In a similar way, the materials dielectric response is also affected by the piezoelectric effect [21]. Considering that the material, with dielectric constant $\varepsilon$, is placed between two electrodes and an external electric field is applied, a surface charge density $\sigma$ will be generated due to the displacement of the electric charges towards the electrodes, with a magnitude $D=\varepsilon . E$. In the case that the material is piezoelectric, the external electric field will also produce a strain, represented in eq.(2). The produced strain can be positive or negative considering the direction of the external electric field with respect to the polarization direction. As mentioned before, an external electric field with the same direction of the polarization direction generates a positive strain, meaning the material expands in this direction. This expansion results in an electric potential with opposite polarity to the polarization direction, meaning that the surface charge density increases and the polarization increases as well. Consequently, by maintaining constant the electric field, the additional polarization increases the displacement of the free charges by a magnitude of $\sigma_{p e}=P_{p e}$ and the total electric displacement can be written as

$$
D=\varepsilon \cdot E+P_{p e}=\varepsilon \cdot E+e \cdot d \cdot E=\bar{\varepsilon} \cdot E
$$

with $\bar{\varepsilon}$ being the effective dielectric constant. 
The piezoelectric effect is a coupling between the elastic variables, $T$ and $S$, and the dielectric ones, D and E [23]. The linear tensor relations between these variables can be given as

$$
\begin{gathered}
S_{p}=s_{p q}^{E} T_{q}+d_{p k} E_{k} \\
D_{i}=d_{i q} T_{q}+\varepsilon_{i k}^{T} E_{k}
\end{gathered}
$$

With $s_{p q}^{E}$ being the elastic compliance tensor at a constant electric field, $\varepsilon_{i k}^{T}$ being the dielectric constant tensor at a constant stress, $d_{k p}$ being the piezoelectric constant tensor, $S_{p}$ the mechanical strain in $p$ direction, $D_{i}$ the electric displacement in the $i$ direction, $T_{q}$ the mechanical stress in the $q$ direction and $E_{k}$ the electric field in the k direction. In the cases of semicrystalline and amorphous polymers and polymer composites [25], the directions are commonly labelled as shown in Figure 5.

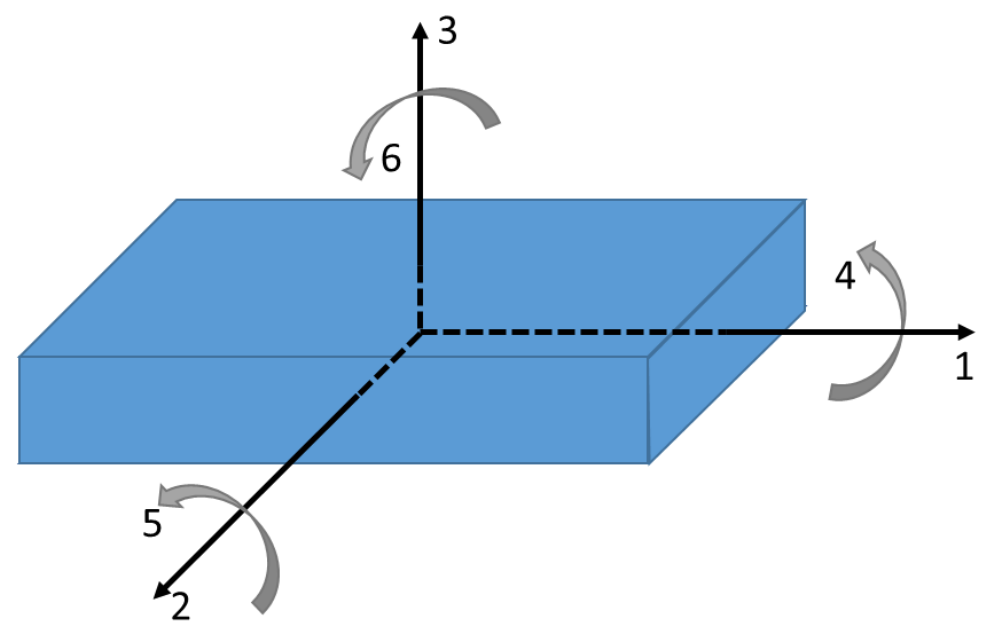

Figure 5 - Tensor directions for mechanical and elastic relations in semicrystalline and amorphous piezoelectric polymer and polymer composites.

For the specific case of PVDF, the most investigated and used piezoelectric polymer, the axis 1 corresponds to the draw or stretch direction, axis 2 corresponds to the transverse direction and axis 3 to the thickness or polarization axis [26]. Using this relation in eqs. 8 and 9 they can be given by 


$$
\begin{aligned}
& {\left[\begin{array}{l}
S_{1} \\
S_{2} \\
S_{3} \\
S_{4} \\
S_{5} \\
s_{6}
\end{array}\right]=\left[\begin{array}{llllll}
s_{11}^{E} & s_{12}^{E} & s_{13}^{E} & s_{14}^{E} & s_{15}^{E} & s_{16}^{E} \\
s_{21}^{E} & s_{22}^{E} & s_{23}^{E} & s_{24}^{E} & s_{25}^{E} & s_{26}^{E} \\
s_{31}^{E} & s_{32}^{E} & s_{33}^{E} & s_{34}^{E} & s_{35}^{E} & s_{36}^{E} \\
s_{41}^{E} & s_{42}^{E} & s_{43}^{E} & s_{44}^{E} & s_{45}^{E} & s_{46}^{E} \\
s_{51}^{E} & s_{52}^{E} & s_{53}^{E} & s_{54}^{E} & s_{55}^{E} & s_{56}^{E} \\
s_{61}^{E} & s_{62}^{E} & s_{63}^{E} & s_{64}^{E} & s_{65}^{E} & s_{66}^{E}
\end{array}\right]\left[\begin{array}{l}
T_{1} \\
T_{2} \\
T_{3} \\
T_{4} \\
T_{5} \\
T_{6}
\end{array}\right]+\left[\begin{array}{lll}
d_{11} & d_{12} & d_{13} \\
d_{21} & d_{22} & d_{23} \\
d_{31} & d_{32} & d_{33} \\
d_{41} & d_{42} & d_{43} \\
d_{51} & d_{52} & d_{53} \\
d_{61} & d_{62} & d_{63}
\end{array}\right]\left[\begin{array}{l}
E_{1} \\
E_{2} \\
E_{3}
\end{array}\right]} \\
& {\left[\begin{array}{l}
D_{1} \\
D_{2} \\
D_{3}
\end{array}\right]=\left[\begin{array}{llllll}
d_{11} & d_{12} & d_{13} & d_{14} & d_{15} & d_{16} \\
d_{21} & d_{22} & d_{23} & d_{24} & d_{25} & d_{26} \\
d_{31} & d_{32} & d_{33} & d_{34} & d_{35} & d_{36}
\end{array}\right]\left[\begin{array}{l}
T_{1} \\
T_{2} \\
T_{3} \\
T_{4} \\
T_{5} \\
T_{6}
\end{array}\right]+\left[\begin{array}{lll}
\varepsilon_{11}^{T} & \varepsilon_{12}^{T} & \varepsilon_{13}^{T} \\
\varepsilon_{21}^{T} & \varepsilon_{22}^{T} & \varepsilon_{23}^{T} \\
\varepsilon_{31}^{T} & \varepsilon_{32}^{T} & \varepsilon_{33}^{T}
\end{array}\right]\left[\begin{array}{l}
E_{1} \\
E_{2} \\
E_{3}
\end{array}\right]}
\end{aligned}
$$

According to eqs. 8 and 9, there are 18 possibilities to couple the electrical and mechanical components of a piezoelectric material with each possibility belonging to one of four possible operating modes. The four main operating modes are known as longitudinal (L), longitudinal shear $\left(\mathrm{S}_{\mathrm{L}}\right)$, transverse $(\mathrm{T})$ and transverse shear $\left(\mathrm{S}_{\mathrm{T}}\right)$, as shown in Figure 6 .

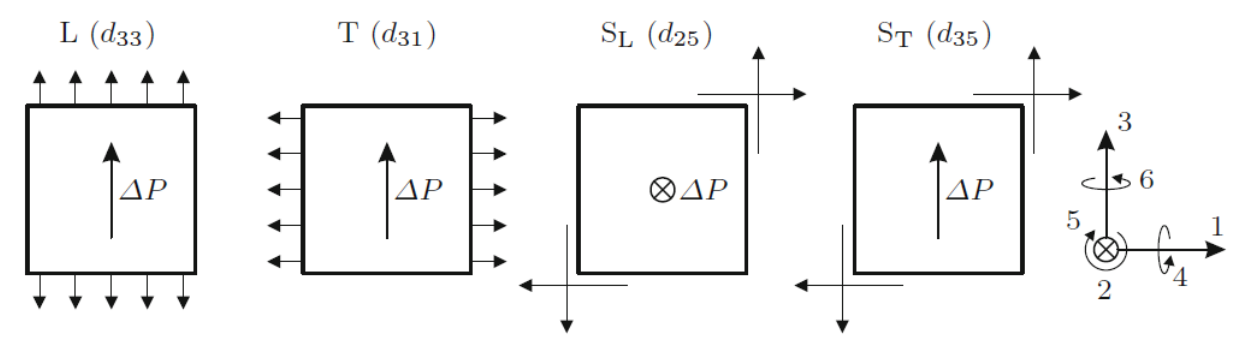

Figure 6 - Schematic representation of four operating modes of the piezoelectric effect. $\Delta P$ is the macroscopic change of the electric polarization [23].

As indicated before and considering the direct piezoelectric effect, a mechanical stress will lead to an electric flux density and by consequence a macroscopic change in the material's polarization in a particular direction will occur [23]. These operating modes and equivalent changes in polarization are described in Table1. 
Table 1 - Direct piezoelectric operating modes and brief description.

\begin{tabular}{|l|l|}
\hline \multicolumn{1}{|c|}{ Operating mode } & \multicolumn{1}{c|}{ Description } \\
\hline Longitudinal mode, $L\left(d_{11}, d_{22}\right.$ and $\left.d_{33}\right)$ & $\begin{array}{l}\text { Application of a normal stress } \\
\text { accompanied by a change in the same } \\
\text { direction of the electric polarization }\end{array}$ \\
\hline $\begin{array}{l}\text { Transverse mode, } T\left(d_{12}, d_{13}, d_{21}, d_{23},\right. \\
\left.d_{31} \text { and } d_{32}\right)\end{array}$ & $\begin{array}{l}\text { Change in electric polarization } \\
\text { perpendicular to the mechanical load }\end{array}$ \\
\hline $\begin{array}{l}\text { Longitudinal shear mode, } S_{L}\left(d_{14}, d_{35} \text { and }\right. \\
d_{36}\end{array}$ & $\begin{array}{l}\text { Application of a shear stress accompanied } \\
\text { by an electric polarization change } \\
\text { perpendicular to the plane of the shearing } \\
\text { stress. }\end{array}$ \\
\hline $\begin{array}{l}\text { Transverse shear mode, } S_{T}\left(d_{15}, d_{16}, d_{24},\right. \\
\left.d_{26}, d_{34} \text { and } d_{35}\right)\end{array}$ & $\begin{array}{l}\text { Change in electric polarization in the } \\
\text { plane of the shearing stress }\end{array}$ \\
\hline
\end{tabular}

Finally, the electromechanical coupling factor is an essential parameter, quantifying the capacity of a material to convert mechanical into electrical energy and vice-versa [23] and can be expressed as

$$
k^{2}=\frac{\text { Converted electrical energy }}{\text { Input mechanical energy }}=\frac{\text { Converted mechanical energy }}{\text { Input electrical energy }}
$$




\section{Piezoelectric composites: definition and types}

Piezoelectric composites belong to the class of smart materials and typically consist of a piezoelectric ceramic filler incorporated in a piezoelectric polymer matrix. Other fillers, such as conductive and magnetic fillers, are also added to these composites, the former just for small amounts of filler as it is shown in figure 7 . Together with those particulate composites, laminated composites, in which the materials are prepared in a layered assembly, are also often presented in the literature and implemented into applications.

Figure 7 shows a schematic representation of a piezoelectric composite with fillers dispersed within the polymer matrix.

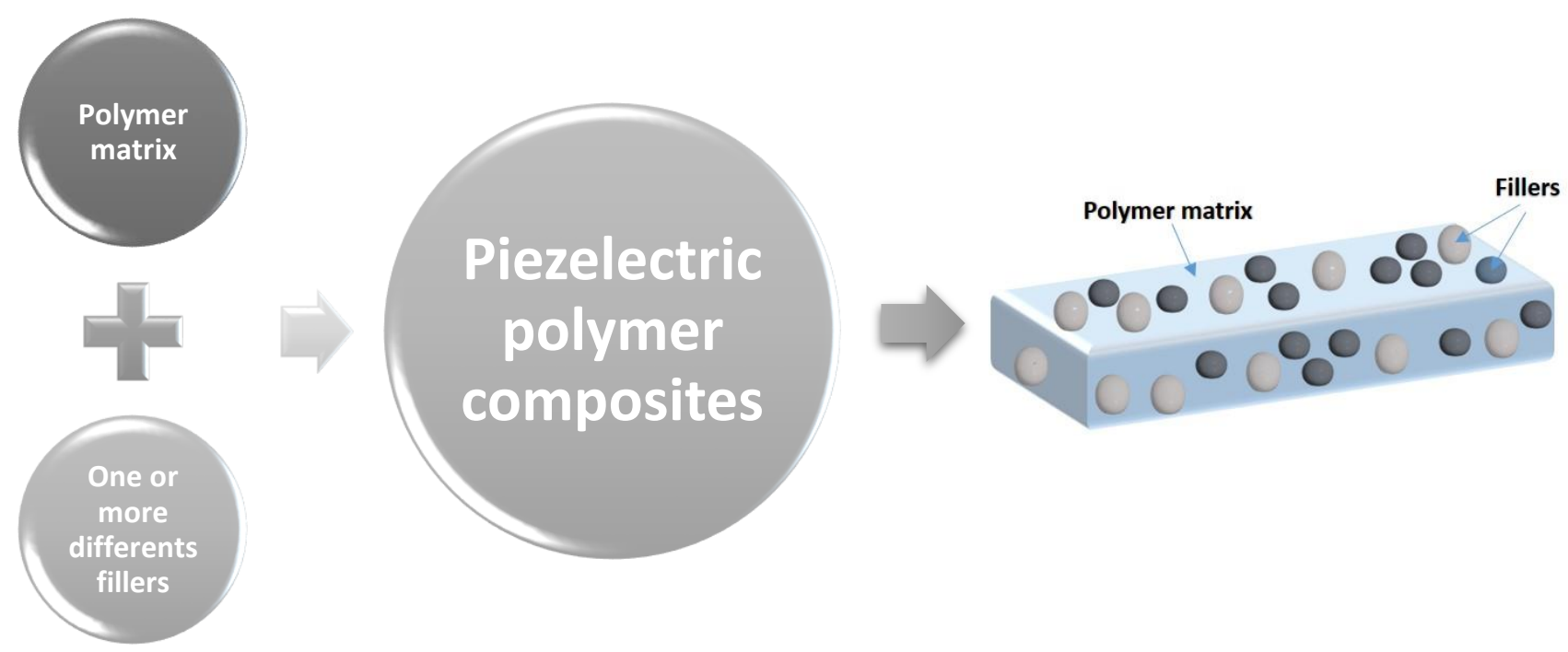

Figure 7 - Schematic representation of a particulate piezoelectric composites.

Polymer composite materials are characterized by low density, flexibility, excellent mechanical properties and thermal stability and higher dielectric and piezoelectric coefficient values than the pristine polymers, which result from the combination of the properties of the polymer matrix and ceramic filler [11, 23].

\subsection{Polymer matrix}

Several piezoelectric polymer matrices are reported in the literature, as shown in Table 2, together with the dielectric value and a relevant piezoelectric coefficient, , and are divided into amorphous and semicrystalline polymers [27].

In the case of semicrystalline polymers, the piezoelectricity is related with crystalline phase in which the dipolar moments can be oriented by the application of electric field [28]. 
Poly (vinylidene fluoride) (PVDF) is a semi-crystalline polymer that stands out in comparison to other semi-crystalline piezoelectric polymers, such as nylon-9, polyureas, poly-L-lactic acid (PLLA), poly (b-hydroxybutyrate) (PHB), among others, due to the high value of the piezoelectric coefficient ( $\mathrm{d} 33 \sim-30 \mathrm{pC} / \mathrm{N})[21,27]$.

PVDF can crystallize in at least four polymorphs known as $\alpha, \beta, \delta$ and $\gamma$-phases, but the crystalline phase with best ferroelectric and piezoelectric properties is the $\beta$-phase. Commonly, $\beta$-phase films are obtained by stretching $\alpha$-phase films at temperatures between $70^{\circ} \mathrm{C}$ and $100{ }^{\circ} \mathrm{C}$ and for stretch ratios from 2 until 5 and also through the addition of various fillers (magnetic: $\mathrm{CoFe}_{2} \mathrm{O}_{4}$, ceramic: $\mathrm{BaTiO}_{3}$, ionic liquids:[EMIM][TFSI]) [29-33]. PVDF electroactive phase content and degree of crystallininty are influenced by the processing conditions, inclusing stretching ratio and temperature, as well as filler type and content [29, 31], which in turn will affect the electroactive properties of the polymer.

Table 2 - Dielectric constant and piezoelectric coefficient for representative semicrystalline and amorphous polymers, respectively.

\begin{tabular}{|c|c|c|c|}
\hline Polymer & Dielectric constant & Piezoelectric coefficient at room temperature & Ref \\
\hline PVDF & $8-12$ & $\mathrm{~d}_{31}=16 \mathrm{pC} / \mathrm{N}$ & {$[28]$} \\
& & $\mathrm{d}_{32}=3 \mathrm{pC} / \mathrm{N}$ & \\
& & $\mathrm{d}_{33}=-20--23 \mathrm{pC} / \mathrm{N}$ & {$[34]$} \\
\hline Nylon-9 & $\sim 3.5$ & $\mathrm{~d}_{31}=1.1 \mathrm{pC} / \mathrm{N}$ & {$[35]$} \\
\hline Polyureas & $2-4$ & $\mathrm{~d}_{31}=10 \mathrm{pC} / \mathrm{N}$ & {$[36]$} \\
\hline PLLA & $2.8-3.5$ & $\mathrm{~d}_{14}=-10 \mathrm{pC} / \mathrm{N}$ & {$[37]$} \\
\hline PHB & $2-3$ & $\mathrm{~d}_{31}=1.58 \mathrm{pC} / \mathrm{N}$ & {$[38]$} \\
\hline Polyimide & 4 & $\mathrm{~d}_{14}=1.3 \mathrm{pC} / \mathrm{N}$ & {$[39]$} \\
\hline PVDC & 3.4 & $\mathrm{~d}_{33}=0.091-0.168 \mathrm{pC} / \mathrm{N}$ & \\
\hline
\end{tabular}

As in the case of amorphous polymer there are no crystalline phases, polarization results in an almost stable state due to the freezing of molecular dipoles. The amorphous polymers most reported in the literature are polyimide [40], polyvinylidene chloride (PVDC) [41], poly(arylene ether nitrile (PAEN) [42], among others. 


\subsection{Ceramic and other filler types}

There are several piezoelectric ceramic materials with high piezoelectric coefficients as shown in table 3, which also are mechanically strong, chemically inert and also show a high dielectric constant [43]. The most used ceramic materials in polymer composites include lead zirconate titanate (PZT), barium titanate $\left(\mathrm{BaTiO}_{3}\right)$, zinc oxide $(\mathrm{ZnO})$, and lead-free as potassium niobate $\left(\mathrm{KNN}, \mathrm{K}_{0.5} \mathrm{Na}_{0.5} \mathrm{NbO}_{3}\right)$, among others [44].

Lead zirconate titanate (PZT) is a ceramic material with chemical formula $\mathrm{Pb}\left(\mathrm{Zr}_{\mathrm{x}} \mathrm{Ti}_{1-\mathrm{x}}\right) \mathrm{O}_{3}$ and perovskite crystalline structure [45]. The phase diagram is complex, but one of the most interesting issues is the existence of the so called morphotropic phase boundary (MPB) dividing the ferroelectric region in two parts: rhombohedral crystalline phase region, rich in $\mathrm{Zr}$ atoms and a tetragonal crystalline phase region rich in $\mathrm{Ti}$ atoms. At room temperature, the MPB is placed in the region $\mathrm{Zr} / \mathrm{Ti}=52 / 48[46,47]$. At the MPB the dielectric and piezoelectric response of the ceramic material is the largest.

Considering its high dielectric constant, $\mathrm{BaTiO}_{3}$ is a very used ceramic material in piezoelectric polymer composites, also crystallizing in a perovskite structure [48].

Table 3 - Dielectric constant and piezoelectric coefficient for some representative ceramic materials.

\begin{tabular}{|c|c|c|c|}
\hline Filler & Dielectric constant & Piezoelectric coefficient & Ref \\
\hline PZT & $200-5000$ & $\mathrm{~d}_{33}=100-1000 \mathrm{pC} / \mathrm{N}$ & {$[28]$} \\
\hline BaTiO3 & $1260-1700$ & $\mathrm{~d}_{31}=-78 \mathrm{pC} / \mathrm{N}$ & {$[49]$} \\
& 11 & $\mathrm{~d}_{33}=190 \mathrm{pC} / \mathrm{N}$ & \\
\hline ZnO & 11 & $\mathrm{~d}_{31}=-5.0 \mathrm{pC} / \mathrm{N}$ & {$[50]$} \\
& 250 & $\mathrm{~d}_{33}=5.9 \mathrm{pC} / \mathrm{N}$ & \\
\hline KNN & $\mathrm{d}_{33} \sim 63 \mathrm{pC} / \mathrm{N}$ & {$[51]$} \\
\hline
\end{tabular}

In addition to improved dielectric and piezoelectric properties, polymer composites with $\mathrm{ZnO}$ fillers show additional properties such as photocatalytic behavior [52].

Also, niobium-based piezoelectric ceramics are very interesting due to being lead-free, showing high dielectric and piezoelectric values, crystallizing also as perovskite structures [53]. 
Generally, the combination of ceramic fillers with piezoelectric polymers leads to composite materials with improved thermal and electrical properties without losing the excellent mechanical properties of the polymeric matrix.

Depending on the size of the ceramic particles, it is possible to produce micro or nanocomposites to develop piezoelectric composite materials with the desired properties for applications. Finally, the processing conditions affect their morphology, physical properties, as well as the macro and microscopic response of these materials, printing technologies allowing to produce large-area composite materials at low processing cost [54].

Other interesting composites are magnetoelectric ones [55], which are being investigated for sensors, data memories, energy collectors, antennas or biomedical applications both as particulate or layered composites. Magnetoelectic materials result from the addition of magnetostrictive fillers, such as $\mathrm{Zn}_{0.2} \mathrm{Mn}_{0.8} \mathrm{Fe}_{2} \mathrm{O}_{4}$ (ZMFO) or $\mathrm{CoFe}_{2} \mathrm{O}_{4}$ (CFO), in a piezoelectric polymer matrix, such as poly (vinylindene fluoride trifluoroethylene) (P(VDF-TrFE) [56] in order to obtain magnetic and magnetoelectric response due to the coupling of the magnetostrictive and piezoelectric phases, allowing manipulation of the electrical polarization by a magnetic field or the magnetization by a field electric [57]. 


\section{Applications}

Piezoelectric composites are optimized for specific applications ranging from mechanical structures to electronic devices, for areas including automotive and aerospace to structural health monitoring and biomedicine. Also, prominent applications of these materials are already found in high energy storage capacitors $[2,58]$.

Proper selection of piezoelectric material is an essential parameter, as it directly influences the device's functionality and performance. The piezoelectric response is generally higher in piezoceramics and therefore make them the most commonly used material. However, piezoceramics are naturally brittle, limiting the strain that it can provide or absorb without being damaged. These materials are susceptible to the growth of fatigue cracks when subjected to high frequency cyclical loads. One the other hand, there are piezoelectric polymers which are flexible, acoustically well matched to water and able to be produced in large areas and in a variety of shapes. Nevertheless, the low electromechanical coupling and lower dielectric constant, limit their applications. In this way, composite appears as a way to improve properties when compared to single phase materials, being also able to produce high piezoelectric output performance [59, 60].

\subsection{Electronic applications}

Most periods of technological development have been distinguished by specific materials, such as stone, bronze and iron age. Currently, one of the driving forces of technology is the use of multifunctional materials, piezoelectric materials being a paradigmatic example of this demand, in particular in development of electronic devices $[2,61]$.

In automotive and aerospace applications there is an increasing need for sensors and actuators. Composites based in lead zirconate titanate and PZT embedded in PVDF have been investigated as sensors in application like benders, tire pressure and knock sensor $[62,63]$. Tire pressure sensors based on PZT-PVDF composites are directly bonded to the inner tire [64].

Protection of spacecraft form radiation used in submarines, seismic and geological research are areas in which piezoelectric materials have been implemented [65-68], mainly in acoustic applications. Piezoelectric microphones based on PVDF have been developed to detect sound inside the cochlea, allowing cochlear implants with normally 
occurring sound pressures and frequencies (ear canal pressures $>50-60 \mathrm{~dB}$ SPL and $0.1-$ $10 \mathrm{kHz}$ ) as shown in figure 8 [69]. Piezoelectric Parylene-C (ortho-chloro-p-xylene) polymer has been also used in microphones and actuators applications [70].

(a)

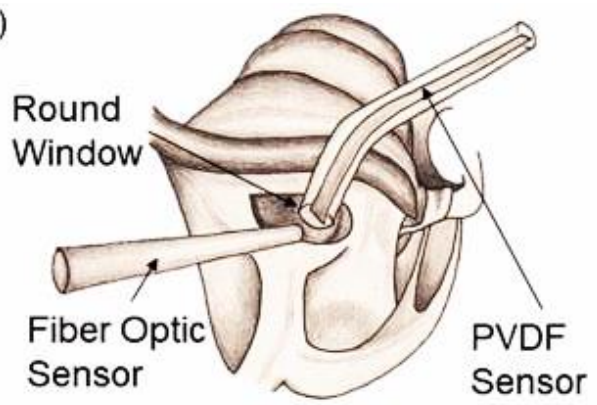

(c)

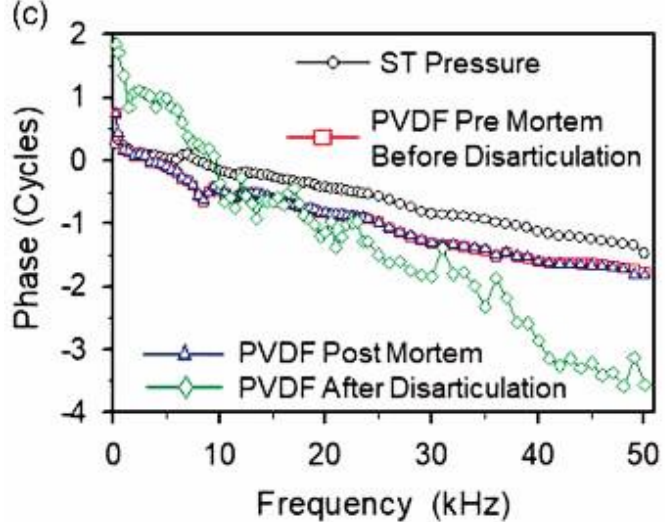

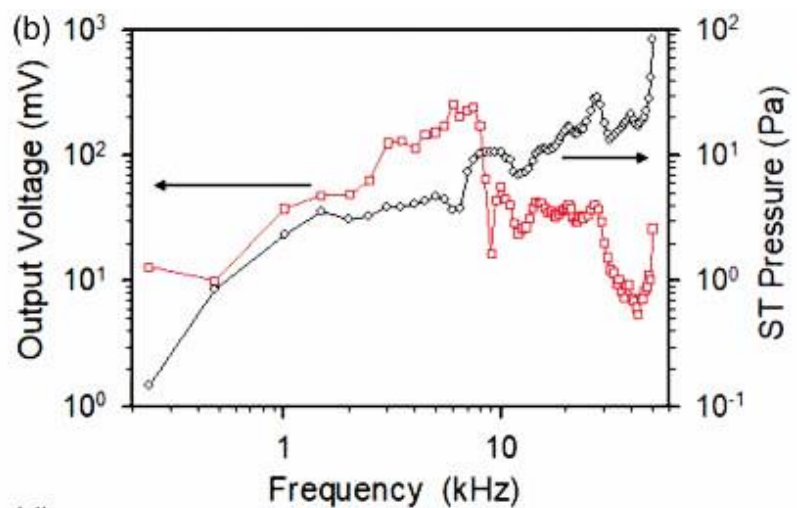

(d)

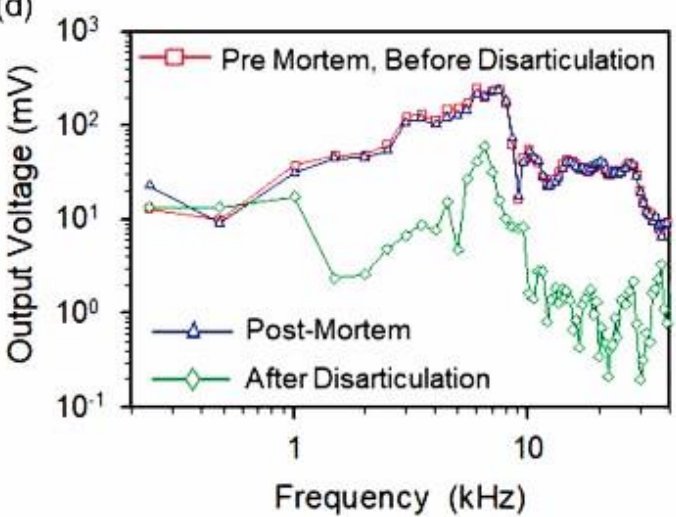

Figure 8 - (a) Schematic representation of fiber optic and PVDF pressure sensor inserted into the round window of a gerbil cochlea. (b) Plot of output voltage (after a gain of 1,000 ) measured with PVDF sensor (red square) and pressure in the scale tympani measured with fiber optic pressure sensor (black circle). (c) Plot of phase measured with fiber optic pressure sensor (black circle), PVDF sensor premortem and before disarticulation (red squares), postmortem (blue triangles), and after disarticulation (greed diamonds). (d) Plot of output voltage (after a gain of 1,000) measured with PVDF sensor premortem or before disarticulation (red squares), postmortem (blue triangles), and after disarticulation (green diamonds) [69].

Currently, with the emergence of mobiles and smart gadgets, as well as wearable electronics and soft robotics, electronic technology has directed new efforts in the development of devices and materials compatible with high touch sensibility and flexible substrates design requirements[71-73]. PVDF and reduced graphene oxide have been described as capable of producing a flexible film with microstructures to mimic the 
epidermal and dermal layer of human fingerprint. This sensor can identify and distinguish between multiple spatiotemporal tactile stimuli including static and dynamic pressure, temperature and vibration with high sensitivities. Thus, in artery vessels, it is possible a precision detection of acoustic sounds and the evaluation of different surface textures can be performed. Wearable devices are an important milestone not only in electronics but also in the field of biomedicine [74]. For the evaluation of structural dynamic strains, flexible nanocomposite sensors using carbon black (CB) fillers and polyvinylidene fluoride (PVDF) matrix was fabricated, the nanocomposite allowing to detect extremely weak strains associated with sources such as structural damage, high-frequency vibration, and ultrasonic waves. This nanocomposite is adequate for strain sensor applications such as advanced bioelectronics, ultrasonic inspection, and in-situ structural health monitoring [75].

A DC current sensor device based on a laminated PVDF / Metglas magnetoelectric composite was developed with the ME coefficient $\left(\alpha_{33}\right)$ of $34.48 \mathrm{~V} \cdot \mathrm{cm}^{-1} \mathrm{Oe}^{-1}$, a linear response $\left(\mathrm{R}^{2}=0.998\right)$ with a sensitivity of $6.7 \mathrm{mV} \cdot \mathrm{A}^{-1}[76]$.

\subsection{Energy harvesting}

Energy harvesting consists in the process of acquiring the surrounding energy of a system and translating it into usable electrical energy.

Piezoelectric transduction is an approach that has received attention in the area of elecromechanical energy harvesting, i.e. the generation of electrical energy from mechanical vibrations [77, 78]. ZnO nanowires [79], lead zirconate titanite (PZT) nanofibers [80, 81], barium titanate $\left(\mathrm{BaTiO}_{3}\right)$ [80, 82, 83] and PVDF [84] are examples of piezoelectric materials that have been used to construct nanogenerators and to effectively power small electronic devices, such as lighting up LEDs [85]. In this context, $\mathrm{FAPbBr}_{3}$ nanoparticles (cubic perovskite structure) uniformly mixed with Polydimethylsiloxane (PDMS) and then spin-coated onto an indium tin oxide (ITO)coated polyethylene terephthalate (PET) substrate and integrated with aluminum (Al), demonstrate a high performance as energy harvesting devices. The FAPbBr3-PDMS composite generates electric potential under an external stress with output voltage and current density of $8.5 \mathrm{~V}$ and $3.8 \mu \mathrm{A} . \mathrm{cm}^{-2}$, respectively, the nanoparticles serving as the energy generation sources as shown in figure 9. The generated energy can be used to charge a capacitor and light up a LED through a bridge rectifier [86]. 

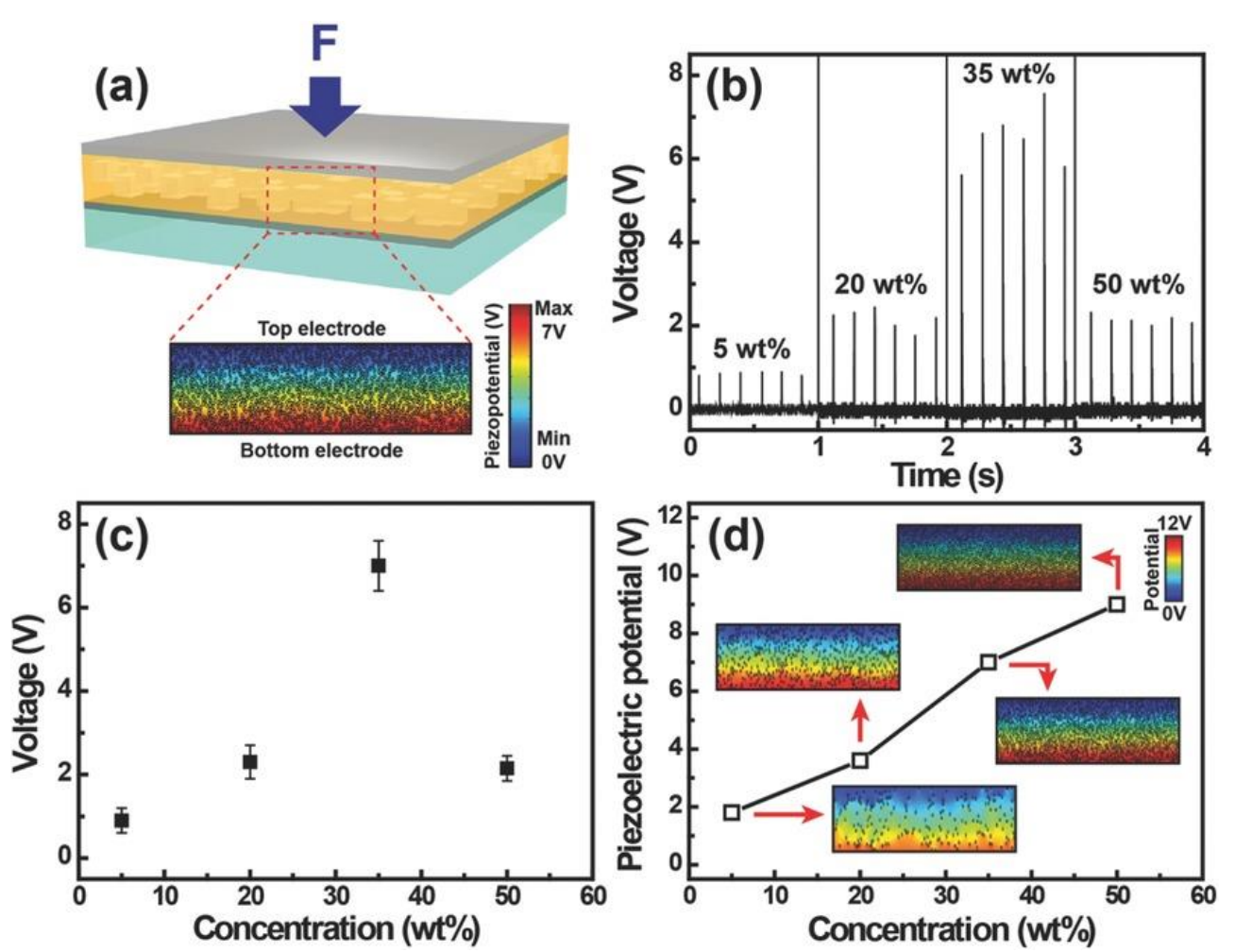

Figure 9 - a) COMSOL simulation model of a nanogenerator. The simulated piezoelectric potential distribution inside the composite between top and bottom electrodes is indicated by color code. b) Output voltage from nanogenerators with different $\mathrm{FAPbBr}_{3}$ nanoparticles concentration. c) Variation of the output voltage with different $\mathrm{FAPbBr}_{3}$ nanoparticles concentrations. d) COMSOL simulation result of output potential distribution of the nanogenerator with different $\mathrm{FAPbBr}_{3}$ nanoparticles concentration [86].

A primary motivation for self-charging structures is to use them for powering small electronic components. Piezoelectric shoes, electronic skin and other energy harvesting devices have been developed to take advantage of the produced vibration from human body activities such as: walking, running, breathing, and dancing to power-up low power electronic devices [87].

The possibility of harnessing the energy lost from a biological activity to provide energy for low-powered electronic devices has been also explored. Cardiac and lung motions serve as inexhaustible sources of energy during the lifespan. One of the first highlights in this area [88] is related with the use of an implantable physiological power supply using PVDF films. The prototype, that used the energy expended for breathing, was implanted in vivo on a mongrel dog and demonstrated a peak voltage of $18 \mathrm{~V}$, which corresponds to a power of about $17 \mathrm{~mW}$. Since then, there are several proposals for such 
devices that translate heartbeat vibrations into electrical energy using piezoelectric composites also for directly powering a cardiac pacemaker by harvesting the kinetic energy of heartbeat [89]. Briefly, $\mathrm{Pb}\left(\mathrm{Mg}_{1 / 3} \mathrm{Nb}_{2 / 3}\right) \mathrm{O}_{3}-(28 \%)-\mathrm{PbTiO}_{3}(\mathrm{PMN}-\mathrm{PT})$ was employed as piezoelectric layer and each side was sputtered with $\mathrm{Cr} / \mathrm{Au}$. A berylliumbronze foil was used to provide uniform stress distribution to the piezoelectric layer and then a PDMS film was deployed by spin-coating. To further improve the stability of the device and avoid potential erosion in the in vivo environment, a parylene film was deposited onto the PDMS film to form a compact and holefree coating layer. In vivo, a commercial cardiac pacemaker was directly powered by the implantable piezoelectric energy generator and monitored its behavior. It was concluded that patients do not need surgical replacement, or at least, the battery replacement will be less frequent. Currently, one of the most important application of energy harvesters is powering implantable biomedical devices [90].

\subsection{Environmental sensors}

Industrialization is causing serious problems in the environment. The accumulation of contaminants in air, soil and water is a threat that instigates the science attention. A very important environmental challenge is water remediation [77], knowing this, physical adsorption, biological methods and chemical oxidation have been applied worldwide [91, 92].

Photocatalytic oxidation has been explored for environmental treatment and purification and coupled with this, piezocatalyses arises. The electric field generated by piezoelectric materials separates free electrons-holes pairs, which further react with dissolved oxygen molecules and water to decompose organic pollutants [93]. $\mathrm{ZnO}, \mathrm{MoS}_{2}$, $\mathrm{Pb}\left(\mathrm{Zr}_{0.52} \mathrm{Ti}_{0.48}\right) \mathrm{O}_{3}, \mathrm{NaNbO}_{3}$, and $\mathrm{BaTiO}_{3}$ have been extensively investigated. It has been demonstrated the applicability of $\mathrm{MoS}_{2} / \mathrm{PDMS}$ nanocomposite as piezocatalyst. The $\mathrm{MoS}_{2} / \mathrm{PDMS}$ film was used as negative layer, while a copper thin layer acted as a positive electrode, the triboelectric nanogenerator was posteriorly fabricated for energy harvesting by hydropower. The piezoelectric part exhibited catalytically active surface on the active edge sites forming free radical oxygen to decompose pollutants. Besides the composite acting as piezocatalyst and energy harvester, it could be utilized as an active sensor for the monitoring of flowing water and its contamination [94]. 
Atmospheric monitoring with specific sensors for organic and inorganic pollutants, potentially toxic elements, and pathogens contributes to the sustainable development of society. Traditional analytical approaches for pollutants monitoring include various chromatographic techniques. However, the response times and high mass sensitivity of a piezoelectric resonator leads to the application of chemical sensors for detecting components (ions, molecules, their fragments or clusters) [95].

A different type of environmental monitoring, although an application with added value, is the use of PVDF fibers with potential to detect stress and strain in the fluid flow, including oceanic current monitoring [96].

\subsection{Biomedical applications}

One of the main effort in the biomedical area is in the development of measuring equipment and health monitoring devices that seek to improve the life quality [97]. Electronic skins or e-skin have received considerable attention in recent years for being a platform for continuous and real-time monitoring of human physiological signals. It finds potential in prosthetics, robots, wearable devices, artificial intelligence, medical equipment, and many other areas. Piezoelectric materials play a significant role in this field $[98,99]$.

Owing to their properties - flexibility, biosafety, easy process -, ZnO, PVDF and $\mathrm{BaTiO}_{3}$ are good candidates for electronic skin applications. In a study using composite nanogenerator based on PVDF fibers, it was verify that the device can be used as a sensor for real-time monitoring of radial artery pulse and respiratory information [100]. Also, $\mathrm{PVDF}_{-T i O}$ nanofibers shown its potential applications in wearable healthcare monitoring systems and the ability of self-cleaning, since $\mathrm{TiO}_{2}$ can efficiently degrade organic pollutants[101]. A device for human motion monitoring was developed based on PVDF nanofibers with tetragonal-phase $\mathrm{BaTiO}_{3} \mathrm{NWs}$ and a wireless circuit system [102]. This device allows that signals from human movement are transmitted wirelessly and displayed in a mobile phone over a long distance $(8 \mathrm{~m})$. The results show the potential in wearable medical electronics in the fields of rehabilitation and sports medicine [102].

When properly processed, piezoelectric materials represent a powerful biomaterial that, in addition to being used as bioelectronic and biomechanical monitoring devices, can interact strictly with biological tissues[103]. Tissue engineering, which is an 
engineering branch attempting to mimic, in vitro or in vivo, cellular microenvironments through scaffolds systems, has also taken advantage of the piezoelectric potential. Piezoelectric materials are used in the production of scaffolds since they reproduce the electrical and mechanical cues existing in the tissues. Thus, piezoelectric scaffolds act as actuators in cellular behavior to promote natural tissue formation [104]. PVDF and its copolymers have already proven their potential in bone [105], skeletal muscle [106] and neural [107] tissue engineering . Furthermore, it was confirmed that the incorporation of different fillers, such as cobalt ferrites $\left(\mathrm{Co}_{2} \mathrm{FO}_{4}\right)$, magnetite $\left(\mathrm{Fe}_{3} \mathrm{O}_{4}\right)$ or Terfenol-D, in PVDF matrix or silk fibroin matrix, ensure the natural regeneration of bones $[108,109]$. Another study with a copolymer of PVDF, P(VDF-TrFE) revealed that the incorporation of $\mathrm{ZnO}$ nanoparticles promote blood vessel formation (angiogenesis) - one of the main problems in tissue engineering approaches. This study also conclude that a composite scaffold favored its integration into the surrounding tissue when compared with noncomposite scaffold [110].

In summary, taking into account their versatility, piezoelectric polymer composites are used in several applications from electronics to biomedical applications, table 4 showing representative composites in different application areas.

Table 4 - Representative piezoelectric polymer composites for different application

\begin{tabular}{|c|c|c|c|}
\hline Composite & Functional characteristic & Application & Ref \\
\hline PVDF-TrFE/ZnO & Pressure sensing & Sensors & {$[111]$} \\
\hline $\begin{array}{c}\text { Polydopamine (PDA)-modified } \\
\text { BaTiO3/PVDF }\end{array}$ & Pressure sensing & Sensors & {$[112]$} \\
\hline $\begin{array}{c}\text { PVDF/polyamide(PA6)/BaTiO3 } \\
\text { PVDF-TrFE/ single-walled } \\
\text { carbon nanotubes (SWCNTs) }\end{array}$ & $\begin{array}{c}\text { Triboelectric nano- } \\
\text { generator }\end{array}$ & Energy harvesting & {$[113]$} \\
\hline Sn3O4/PVDF & Photocatalytic & Energy harvesting & {$[114]$} \\
\hline Ag-TiO2/PVDF-HFP & $\begin{array}{c}\text { Photocatalytic and } \\
\text { antimicrobial }\end{array}$ & Environmental & {$[115]$} \\
\hline PVDF/Graphene oxide (GO) & Electrical stimulation & Biomedical & {$[117]$} \\
\hline Graphene(G)/ BaTiO3/PMMA & Piezoelectricity & Biomedical & {$[118]$} \\
\hline
\end{tabular}




\section{Conclusion}

Piezoelectric polymer composite materials are a class of materials that belong to smart and multifunctional materials. The ability to transduce mechanical to electrical signals and vice-versa provide their materials with increasing technological interest for the development of sensors and actuators or energy harvesters in the form of thin, flexible and potentially large area films. This material class combines the excellent properties of ceramic fillers and polymeric matrix, allowing high dielectric constant and piezoelectric coefficient and excellent thermal and mechanical properties, being successfully implemented in areas such as consumer electronics, aerospace and automotive applications or biomedicine.

Future trends are the continuous development of multifunctional tricomposites with two different fillers, the use of environmental friendlier materials, the precise tuning of control material properties for specific applications and to improve integration into devices by techniques such as additive manufacturing techniques.

\section{Acknowledgments}

The authors thank the FCT (Fundação para a Ciência e Tecnologia) for financial support under the framework of Strategic Funding grants UID/FIS/04650/2020, UID/EEA/04436/2020 and UID/QUI/0686/2020; and project no. PTDC/FISMAC/28157/2017， PTDC/BTM-MAT/28237/2017 and PTDC/EMDEMD/28159/2017. The authors also thank the FCT for financial support under grants SFRH/BD/145455/2019 (E.C.), SFRH/BD/145345/2019 (L.F.) and SFRH/BPD/112547/2015 (C.M.C.). Financial support from the Basque Government Industry and Education Departments under the ELKARTEK, HAZITEK and PIBA (PIBA-2018-06) programs, respectively, is also acknowledged.

\section{References}

1. Baur, C., et al., Advances in Piezoelectric Polymer Composites for Vibrational Energy Harvesting, in Polymer Composites for Energy Harvesting, Conversion, and Storage. 2014, American Chemical Society. p. 1-27.

2. Akdogan, E.K., M. Allahverdi, and A. Safari, Piezoelectric composites for sensor and actuator applications. IEEE Transactions on Ultrasonics, Ferroelectrics, and Frequency Control, 2005. 52(5): p. 746-775. 
3. Gong, H., et al., Preparation and properties of cement based piezoelectric composites modified by CNTs. Current Applied Physics, 2011. 11(3): p. 653-656.

4. Tatarenko, A.S., et al., Microwave magnetoelectric effects in ferrite-piezoelectric composites and dual electric and magnetic field tunable filters. Journal of Electroceramics, 2010. 24(1): p. 5-9.

5. Taunaumang, H., I.L. Guy, and H.L.W. Chan, Electromechanical properties of 1-3 piezoelectric ceramic/piezoelectric polymer composites. Journal of Applied Physics, 1994. 76(1): p. 484-489.

6. Firmino Mendes, S., et al., Effect of the ceramic grain size and concentration on the dynamical mechanical and dielectric behavior of poly(vinilidene fluoride)/Pb(Zr0.53Ti0.47)O3 composites. Applied Physics A, 2009. 96(4): p. 899908.

7. Dumoulin, C. and A. Deraemaeker, A study on the performance of piezoelectric composite materials for designing embedded transducers for concrete assessment. Smart Materials and Structures, 2018. 27(3): p. 035008.

8. Khanbareh, H., V.Y. Topolov, and C.R. Bowen, Piezo-Particulate Composites: Manufacturing, Properties, Applications. 2019: Springer International Publishing.

9. Kapuria, S., P. Kumari, and J.K. Nath, Efficient modeling of smart piezoelectric composite laminates: a review. Acta Mechanica, 2010. 214(1): p. 31-48.

10. Newnham, R.E., D.P. Skinner, and L.E. Cross, Connectivity and piezoelectricpyroelectric composites. Materials Research Bulletin, 1978. 13(5): p. 525-536.

11. Uchino, K., Piezoelectric composite materials. Advanced Piezoelectric Materials: Science and Technology, 2010: p. 318-346.

12. Singh, H.H., S. Singh, and N. Khare, Design of flexible PVDF/NaNbO3/RGO nanogenerator and understanding the role of nanofillers in the output voltage signal. Composites Science and Technology, 2017. 149: p. 127-133.

13. Ren, L., et al., Co-continuous structural polystyrene/poly(vinylidene fluoride) nanocomposites with high dielectric constant and magnetic properties. Composites Communications, 2017. 4: p. 24-32.

14. Chen, T. and B. Liu, Enhanced dielectric properties of poly(vinylidene fluoride) composite filled with polyaniline-iron core-shell nanocomposites. Materials Letters, 2018. 210: p. 165-168.

15. Hankel, W.G., Uber die aktinound piezoelektrischen eigenschaften des bergkrystalles und ihre beziehung zu den thermoelektrischen. Abh. Sächs, 1881. 12: p. 457.

16. Curie, J. and P. Curie, Phénomènes électriques des cristaux hémièdres à faces inclinées. J. Phys. Theor. Appl., 1882. 1(1): p. 245-251.

17. Lippmann, G., Principe de la conservation de l'électricité, ou second principe de la théorie des phénomènes électriques. J. Phys. Theor. Appl., 1881. 10(1): p. 381-394.

18. Tichý, J., et al., Fundamentals of Piezoelectric Sensorics: Mechanical, Dielectric, and Thermodynamical Properties of Piezoelectric Materials. 2010: Springer Berlin Heidelberg.

19. Kawai, H., The Piezoelectricity of Poly (vinylidene Fluoride). Japanese Journal of Applied Physics, 1969. 8(7): p. 975-976.

20. Dahiya, R.S. and M. Valle, Robotic Tactile Sensing: Technologies and System. 2014: Springer Publishing Company, Incorporated.

21. Arnau, A. and D. Soares, Fundamentals of Piezoelectricity, in Piezoelectric Transducers and Applications, A.A. Vives, Editor. 2008, Springer Berlin Heidelberg: Berlin, Heidelberg. p. 1-38.

22. Pinna, L., et al. Analysis of self-powered vibration-based energy scavenging system. in 2010 IEEE International Symposium on Industrial Electronics. 2010.

23. Rupitsch, S.J., Piezoelectric Sensors and Actuators: Fundamentals and Applications. 2018: Springer Berlin Heidelberg.

24. ANSI/IEEE, IEEE Standard on Piezoelectricity. IEEE Transactions on Sonics and Ultrasonics, 1984. 31(2): p. i-i. 
25. Vinogradov, A., Constitutive model of piezoelectric polymer PVDF. 1999 Symposium on Smart Structures and Materials. Vol. 3667. 1999: SPIE.

26. Broadhurst, M.G. and G.T. Davis, Physical basis for piezoelectricity in PVDF. Ferroelectrics, 1984. 60(1): p. 3-13.

27. Harrison, J.S., Piezoelectric Polymers. 2001.

28. Heywang, W., K. Lubitz, and W. Wersing, Piezoelectricity: Evolution and Future of a Technology. 2008: Springer Berlin Heidelberg.

29. Sencadas, V., R. Gregorio, and S. Lanceros-Méndez, $\alpha$ to $\beta$ Phase Transformation and Microestructural Changes of PVDF Films Induced by Uniaxial Stretch. Journal of Macromolecular Science, Part B: Physics, 2009. 48(3): p. 514 - 525.

30. Branciforti, M.C., et al., New technique of processing highly oriented poly(vinylidene fluoride) films exclusively in the beta phase. Journal of Polymer Science Part B: Polymer Physics, 2007. 45(19): p. 2793-2801.

31. V. Sencadas, et al., $\alpha$ - to $\beta$ Transformation on PVDF Films Obtained by Uniaxial Stretch. Materials Science Forum, 2006. 514 - 516: p. 872-876.

32. Martins, P., A.C. Lopes, and S. Lanceros-Mendez, Electroactive phases of poly(vinylidene fluoride): Determination, processing and applications. Progress in Polymer Science, 2014. 39(4): p. 683-706.

33. Ribeiro, C., et al., Electroactive poly(vinylidene fluoride)-based structures for advanced applications. Nature Protocols, 2018. 13(4): p. 681-704.

34. Wu, G., O. Yano, and T. Soen, Dielectric and Piezoelectric Properties of Nylon 9 and Nylon 11. Polymer Journal, 1986. 18(1): p. 51-61.

35. Hattori, T., et al., Piezoelectric and ferroelectric properties of polyurea-5 thin films prepared by vapor deposition polymerization. Journal of Applied Physics, 1996. 79(3): p. 1713-1721.

36. Bernard, F., et al., Direct Piezoelectric Coefficient Measurements of PVDF and PLLA under Controlled Strain and Stress. Proceedings, 2017. 1(4): p. 335.

37. Fukada, E. and Y. Ando, Piezoelectric properties of poly- $\beta$-hydroxybutyrate and copolymers of $\beta$-hydroxybutyrate and $\beta$-hydroxyvalerate. International Journal of Biological Macromolecules, 1986. 8(6): p. 361-366.

38. Gonzalo, B., et al., Dielectric Properties of Piezoelectric Polyimides. Ferroelectrics, 2008. 370(1): p. 3-10.

39. Mopsik, F.I. and M.G. Broadhurst, Molecular dipole electrets. Journal of Applied Physics, 1975. 46(10): p. 4204-4208.

40. Ounaies, Z., et al., Dielectric Properties of Piezoelectric Polyimides. MRS Proceedings, 1997. 459.

41. Yue, Z. and J. Economy, 4 - Carbonization and activation for production of activated carbon fibers, in Activated Carbon Fiber and Textiles, J.Y. Chen, Editor. 2017, Woodhead Publishing: Oxford. p. 61-139.

42. Liu, C., et al., Component Adjustment of Poly(arylene ether nitrile) with Sulfonic and Carboxylic Groups for Dielectric Films. Polymers, 2019. 11(7): p. 1135.

43. Schneider, G.A., Fracture of Piezoceramics, in Encyclopedia of Materials: Science and Technology, K.H.J. Buschow, et al., Editors. 2001, Elsevier: Oxford. p. 3319-3321.

44. Dincer, I., Comprehensive Energy Systems. 2018: Elsevier Science.

45. Cross, L.E., Ferroelectric materials for electromechanical transducer applications. Materials Chemistry and Physics, 1996. 43(2): p. 108-115.

46. Amin, A., et al., Phenomenological and structural study of a low-temperature phase transition in the PbZrO3---PbTiO3 system. Journal of Solid State Chemistry, 1981. 37(2): p. 248-255.

47. Wu, A. and et al., Domain populations in lead zirconate titanate thin films of different compositions via piezoresponse force microscopy. Nanotechnology, 2005. 16(11): p. 2587.

48. Hennings, D., Barium titanate based ceramic materials for dielectric use. International Journal of High Technology Ceramics, 1987. 3(2): p. 91-111. 
49. Gallego-Juarez, J.A., Piezoelectric ceramics and ultrasonic transducers. Journal of Physics E: Scientific Instruments, 1989. 22(10): p. 804-816.

50. Fraga, M.A., et al., Wide bandgap semiconductor thin films for piezoelectric and piezoresistive MEMS sensors applied at high temperatures: an overview. Microsystem Technologies, 2014. 20(1): p. 9-21.

51. Dwivedi, S., T. Pareek, and S. Kumar, Structure, dielectric, and piezoelectric properties of K0.5Na0.5NbO3-based lead-free ceramics. RSC Advances, 2018. 8(43): p. 2428624296.

52. Morales-Flores, N., U. Pal, and E. Sánchez Mora, Photocatalytic behavior of ZnO and Pt-incorporated ZnO nanoparticles in phenol degradation. Applied Catalysis A: General, 2011. 394(1): p. 269-275.

53. Kosec, M., et al., KNN-Based Piezoelectric Ceramics, in Piezoelectric and Acoustic Materials for Transducer Applications, A. Safari and E.K. Akdoğan, Editors. 2008, Springer US: Boston, MA. p. 81-102.

54. Zhang, Q., K. Zhang, and G. Hu, Smart three-dimensional lightweight structure triggered from a thin composite sheet via 3D printing technique. Scientific Reports, 2016. 6(1): p. 22431.

55. Martins, P. and S. Lanceros-Méndez, Polymer-based magnetoelectric materials: To be or not to be. Applied Materials Today, 2019. 15: p. 558-561.

56. Martins, P., et al., Tailored Magnetic and Magnetoelectric Responses of Polymer-Based Composites. ACS Applied Materials \& Interfaces, 2015. 7(27): p. 15017-15022.

57. Martins, P. and S. Lanceros-Méndez, Polymer-Based Magnetoelectric Materials. Advanced Functional Materials, 2013. 23(27): p. 3371-3385.

58. Qi, Y., P.K. Purohit, and M.C. McAlpine. Enhanced piezoelectricity and stretchability in energy harvesting devices fabricated from buckled PZT ribbons. in Proceedings of SPIE - The International Society for Optical Engineering. 2011.

59. Yun, B.K., et al., Lead-free linbo3 nanowire-based nanocomposite for piezoelectric power generation. Nanoscale Research Letters, 2014. 9(1): p. 1-7.

60. Schmidt, L.C., et al., Nontemplate synthesis of CH3NH3PbBr3 perovskite nanoparticles. Journal of the American Chemical Society, 2014. 136(3): p. 850-853.

61. Gupta, P. and R. Srivastava, Overview of Multi Functional Materials. 2010.

62. Kulkarni, H., et al. Application of piezoelectric technology in automotive systems. in Materials Today: Proceedings. 2018.

63. Shirinov, A.V. and W.K. Schomburg, Pressure sensor from a PVDF film. Sensors and Actuators, A: Physical, 2008. 142(1): p. 48-55.

64. Van Den Ende, D.A., et al., Direct strain energy harvesting in automobile tires using piezoelectric PZT-polymer composites. Smart Materials and Structures, 2012. 21(1).

65. Rus, G., et al., Damage detection in piezoceramics via BEM, in Key Engineering Materials. 2010. p. 381-384.

66. Parali, L., I. Sabikołlu, and M.A. Kurbanov, Piezoelectric properties of the new generation active matrix hybrid (micro-nano) composites. Applied Surface Science, 2014. 318: p. 6-9.

67. Li, H., Z. Daniel Deng, and T.J. Carlson, Piezoelectric materials used in underwater acoustic transducers. Sensor Letters, 2012. 10(3-4): p. 679-697.

68. Chocat, N., et al. Piezoelectric multimaterial fibers. in Materials Research Society Symposium Proceedings. 2011.

69. Park, S., et al., PVDF-Based Piezoelectric Microphone for Sound Detection Inside the Cochlea: Toward Totally Implantable Cochlear Implants. Trends in Hearing, 2018. 22: p. 2331216518774450.

70. Kim, J.Y.H., et al. Piezoelectric Parylene-C MEMS microphone. in 2013 Transducers and Eurosensors XXVII: The 17th International Conference on Solid-State Sensors, Actuators and Microsystems, TRANSDUCERS and EUROSENSORS 2013. 2013.

71. Deutz, D.B., et al., Piezoelectric Lead Zirconium Titanate Composite Touch Sensors for Integration with Flexible OLED Technology. Ferroelectrics, 2015. 480(1): p. 1-9. 
72. van der Zwaag, S., D.A. van den Ende, and W.A. Groen, Sensing and Energy Harvesting Novel Polymer Composites, in Materials Experience: Fundamentals of Materials and Design. 2013. p. 221-234.

73. Tressler, J.F., S. Alkoy, and R.E. Newnham, Piezoelectric sensors and sensor materials. Journal of Electroceramics, 1998. 2(4): p. 257-272.

74. Park, J., et al., Nanomaterials: Fingertip skin-inspired microstructured ferroelectric skins discriminate static/dynamic pressure and temperature stimuli. Science Advances, 2015. 1(9).

75. $\mathrm{Xu}, \mathrm{H}$., et al., Broadband dynamic responses of flexible carbon black/poly (vinylidene fluoride) nanocomposites: A sensitivity study. Composites Science and Technology, 2017. 149: p. 246-253.

76. Castro, N., et al., Development of a contactless DC current sensor with high linearity and sensitivity based on the magnetoelectric effect. Smart Materials and Structures, 2018. 27(6): p. 065012.

77. Inman, D., Introduction to piezoelectric energy harvesting. Piezoelectric Energy Harvesting, 2011: p. 1-18.

78. Costa, P., et al., Recent Progress on Piezoelectric, Pyroelectric, and Magnetoelectric Polymer-Based Energy-Harvesting Devices. Energy Technology, 2019. 7(7): p. 1800852.

79. Wang, Z.L. and J. Song, Piezoelectric nanogenerators based on zinc oxide nanowire arrays. Science, 2006. 312(5771): p. 242-246.

80. Anton, S.R. and H.A. Sodano, A review of power harvesting using piezoelectric materials (2003-2006). Smart Materials and Structures, 2007. 16(3): p. R1-R21.

81. Chen, X., et al., $1.6 v$ nanogenerator for mechanical energy harvesting using PZT nanofibers. Nano Letters, 2010. 10(6): p. 2133-2137.

82. Park, K.I., et al., Flexible nanocomposite generator made of BaTiO <inf $>3</$ inf $>$ nanoparticles and graphitic carbons. Advanced Materials, 2012. 24(22): p. 29993004.

83. Nunes-Pereira, J., et al., Energy harvesting performance of BaTiO<inf $>3</$ inf $>/$ poly(vinylidene fluoride-trifluoroethylene) spin coated nanocomposites. Composites Part B: Engineering, 2015. 72: p. 130-136.

84. Chang, C., et al., Direct-write piezoelectric polymeric nanogenerator with high energy conversion efficiency. Nano Letters, 2010. 10(2): p. 726-731.

85. Pan, C., et al., High-resolution electroluminescent imaging of pressure distribution using a piezoelectric nanowire LED array. Nature Photonics, 2013. 7(9): p. 752-758.

86. Ding, R., et al., Flexible Piezoelectric Nanocomposite Generators Based on Formamidinium Lead Halide Perovskite Nanoparticles. Advanced Functional Materials, 2016. 26(42): p. 7708-7716.

87. Paulides, J.J.H., et al., Power from the people: Human-powered small-scale generation system for a sustainable dance club. IEEE Industry Applications Magazine, 2011. 17(5): p. 20-26.

88. Häusler, E. and L. Stein, Implantable physiological power supply with PVDF film. Ferroelectrics, 1984. 60(1): p. 277-282.

89. Li, N., et al., Direct Powering a Real Cardiac Pacemaker by Natural Energy of a Heartbeat. ACS Nano, 2019. 13(3): p. 2822-2830.

90. Yang, Z., et al., High-Performance Piezoelectric Energy Harvesters and Their Applications. Joule, 2018. 2(4): p. 642-697.

91. Chawla, S., et al., Zinc peroxide nanomaterial as an adsorbent for removal of Congo red dye from waste water. Ecotoxicology and Environmental Safety, 2017. 135: p. 68-74.

92. Li, X., et al., Highly efficient advanced oxidation processes (AOPs) based on premagnetization $\mathrm{FeO}$ for wastewater treatment. Separation and Purification Technology, 2017. 178: p. 49-55. 
93. Liu, X., et al., Significantly enhanced piezo-photocatalytic capability in BaTiO3 nanowires for degrading organic dye. Journal of Materiomics, 2020.

94. Lin, J.H., et al., Single- and few-layers MoS2 nanocomposite as piezo-catalyst in dark and self-powered active sensor. Nano Energy, 2017. 31: p. 575-581.

95. Kuchmenko, T.A. and L.B. Lvova, A Perspective on Recent Advances in Piezoelectric Chemical Sensors for Environmental Monitoring and Foodstuffs Analysis. Chemosensors, 2019. 7(3): p. 39.

96. Egusa, S., et al., Multimaterial piezoelectric fibres. Nature Materials, 2010. 9(8): p. 643-648.

97. MirHojjat, S., et al., An Overview of Intra-Body Communication Transceivers for Biomedical Applications, in Encyclopedia of Information Science and Technology, Third Edition. 2015, IGI Global: Hershey, PA, USA. p. 469-478.

98. Jason, N.N., M.D. Ho, and W. Cheng, Resistive electronic skin. Journal of Materials Chemistry C, 2017. 5(24): p. 5845-5866.

99. Chen, S., et al., Recent Developments in Graphene-Based Tactile Sensors and E-Skins. Advanced Materials Technologies, 2018.3(2).

100. Chen, X., et al., Flexible fiber-based hybrid nanogenerator for biomechanical energy harvesting and physiological monitoring. Nano Energy, 2017. 38: p. 43-50.

101. Dong, C., et al., Self-powering/self-cleaning electronic-skin basing on PVDF/TiO $<$ inf $>2</$ inf $>$ nanofibers for actively detecting body motion and degrading organic pollutants. Applied Surface Science, 2017. 416: p. 424-431.

102. Guo, W., et al., Wireless piezoelectric devices based on electrospun PVDF/BaTiO3 NW nanocomposite fibers for human motion monitoring. Nanoscale, 2018. 10(37): p. 17751-17760.

103. Chorsi, M.T., et al., Piezoelectric Biomaterials for Sensors and Actuators. Advanced Materials, 2019. 31(1).

104. Ribeiro, C., et al., Piezoelectric polymers as biomaterials for tissue engineering applications. Colloids and Surfaces B: Biointerfaces, 2015. 136: p. 46-55.

105. Ribeiro, C., et al., Proving the suitability of magnetoelectric stimuli for tissue engineering applications. Colloids and Surfaces B: Biointerfaces, 2016. 140: p. 430436.

106. Ribeiro, S., et al., Electroactive biomaterial surface engineering effects on muscle cells differentiation. Materials Science and Engineering C, 2018. 92: p. 868-874.

107. Royo-Gascon, N., et al., Piezoelectric substrates promote neurite growth in rat spinal cord neurons. Annals of Biomedical Engineering, 2013. 41(1): p. 112-122.

108. Brito-Pereira, R., et al., Silk fibroin-magnetic hybrid composite electrospun fibers for tissue engineering applications. Composites Part B: Engineering, 2018. 141: p. 7075.

109. Maciel, M.M., et al., Relation between fiber orientation and mechanical properties of nano-engineered poly(vinylidene fluoride) electrospun composite fiber mats. Composites Part B: Engineering, 2018. 139: p. 146-154.

110. Augustine, R., et al., Electrospun poly(vinylidene fluoride-trifluoroethylene)/zinc oxide nanocomposite tissue engineering scaffolds with enhanced cell adhesion and blood vessel formation. Nano Research, 2017. 10(10): p. 3358-3376.

111. Cherumannil Karumuthil, S., et al., Fabrication of poly (vinylidene fluoridetrifluoroethylene) - Zinc oxide based piezoelectric pressure sensor. Sensors and Actuators A: Physical, 2020. 303: p. 111677.

112. Yang, Y., et al., Flexible piezoelectric pressure sensor based on polydopamine-modified BaTiO3/PVDF composite film for human motion monitoring. Sensors and Actuators A: Physical, 2020. 301: p. 111789.

113. Sun, Z., et al., A Green Triboelectric Nano-Generator Composite of Degradable Cellulose, Piezoelectric Polymers of PVDF/PA6, and Nanoparticles of BaTiO3. Sensors, 2020. 20(2): p. 506. 
114. Shepelin, N.A., et al., Printed recyclable and self-poled polymer piezoelectric generators through single-walled carbon nanotube templating. Energy \& Environmental Science, 2020. 13(3): p. 868-883.

115. Han, S., et al., Assembling Sn304 nanostructures on a hydrophobic PVDF film through metal-F coordination to construct a piezotronic effect-enhanced Sn304/PVDF hybrid photocatalyst. Nano Energy, 2020. 72: p. 104688.

116. Salazar, $\mathrm{H}$., et al., Photocatalytic and antimicrobial multifunctional nanocomposite membranes for emerging pollutants water treatment applications. Chemosphere, 2020. 250: p. 126299.

117. Shuai, C., et al., Graphene oxide assists polyvinylidene fluoride scaffold to reconstruct electrical microenvironment of bone tissue. Materials \& Design, 2020. 190: p. 108564.

118. Tang, Y., et al., Graphene/barium titanate/polymethyl methacrylate bio-piezoelectric composites for biomedical application. Ceramics International, 2020. 46(5): p. 65676574. 